\title{
A Molecule-Based 1:2 Digital Demultiplexer
}

Joakim Andréasson, ${ }^{*} \dagger$ Stephen D. Straight, ${ }^{\ddagger}$ Subhajit Bandyopadhyay, ${ }^{\S}$ Reginald H.

Mitchell, ${ }^{\S}$ Thomas A. Moore, ${ }^{*, \star}$ Ana L. Moore, ${ }^{*, \ddagger}$ and Devens Gust ${ }^{*, \neq}$

Department of Chemical and Biological Engineering, Chalmers University of

Technology, SE-41296 Göteborg, Sweden, Department of Chemistry and Biochemistry,

Arizona State University, Tempe, AZ 85287-1604, USA, and Department of Chemistry,

University of Victoria, P. O. Box 3065, Victoria, BC, Canada V8W 3V6

RECEIVED DATE (to be automatically inserted after your manuscript is accepted if required according to the journal that you are submitting your paper to)

A trichromophoric molecule consisting of a porphyrin linked to both a dihydropyrene and a dihydroindolizine-type photochrome, in combination with a third harmonic generating crystal, functions as a 1:2 digital demultiplexer with photonic inputs and outputs. Each of the two photochromes may be cycled independently between two metastable forms, leading to four photoisomers, three of which are used in the demultiplexer. These isomers interact photochemically with the porphyrin in order to yield the demultiplexer function. With the address input (1064 nm light) off, one output of the device (porphyrin fluorescence) tracks the state of the data input (532 nm light). When the address input is turned on, the second output (absorbance at $572 \mathrm{~nm}$ ) tracks the

\footnotetext{
* Corresponding authors. E-mail a-son@chalmers.se (JA), gust@asu.edu (DG), tmoore@asu.edu (TAM), amoore@asu.edu (ALM.)

Chalmers University of Technology

Arizona State University

$\S$ University of Victoria
} 
state of the data input, while the first output remains off. The demultiplexer does not require chemical or electrical inputs, and can cycle through its operational sequences multiple times.

\section{Introduction}

Photochromic molecules, which can be isomerized back and forth between two metastable forms using light, are well suited to be components of molecule-based binary devices. We have previously shown that by covalently coupling photochromes to other chromophores, with which they can communicate by intramolecular electron or energy transfer, simple molecular switches, ${ }^{1-7}$ Boolean logic gates, ${ }^{8-10}$ and combinations of logic gates in the form of half adders ${ }^{11,12}$ can be realized. ${ }^{13}$ This work and that of others in the area ${ }^{1,14-33}$ has shown that such molecular "devices" can function very well, and may eventually be useful for practical applications (although not necessarily the same ones currently filled by electronic digital computers.) Recently, we were able to demonstrate that molecular triad 1 (Figure 1) can be made to function not only as molecular AND and INHIBIT gates, ${ }^{8}$ but also as a 2:1 digital multiplexer. ${ }^{34}$ The function of a multiplexer is to encode the digital state of each of two or more inputs into a single output through the use of a control input. Thus, a multiplexer can combine several input signals into a single output signal for transmission to a receiver, allowing multiple data streams to be transmitted on a single data line. The receiver must then sort out the entangled data streams from this single signal, directing the data from each of the initial inputs to a separate output. This function is

performed by a demultiplexer. Here, we demonstrate how triad $\mathbf{1}$, in combination with lasers and a third-harmonic-generating (THG) crystal, may be used as a 2:1 digital demultiplexer.

The truth table of the demultiplexer is shown in Table 1. The binary input $(I n)$ can exist in either of two states, on (1) or off (0). Each of the two outputs, $O 1$ and $O 2$ may also have the value 1 or 0 . A second input, the address $(A d)$ or enabler, may likewise be set either on or off. When $A d$ is set off, then output $O 1$ reports the state of the input $I n$ and $O 2$ remains off, whereas when $A d$ is switched on, $O 2$ 
reports the state of $I n$. Thus, if the output of the multiplexer serves as the input of the demultiplexer, and the control input of the multiplexer and the address input of the demultiplexer are switched on and off in synchrony, demultiplexer output $O 1$ will faithfully track the state of one of the multiplexer inputs, whereas demultiplexer output $O 2$ will report the state of the second multiplexer input. Multiplexed data transmission will be achieved.

Table 1. Truth table for the 1:2 demultiplexer

\begin{tabular}{cccc}
\hline $\begin{array}{c}\text { input } I n \\
(532 \mathrm{~nm})\end{array}$ & $\begin{array}{c}\text { input } A d \\
(1064 \mathrm{~nm})\end{array}$ & $\begin{array}{c}\text { output } O 1 \\
\text { (fluorescence) }\end{array}$ & $\begin{array}{c}\text { output } O 2 \\
\text { (absorbance) }\end{array}$ \\
\hline 0 & 0 & 0 & 0 \\
1 & 0 & 1 & 0 \\
0 & 1 & 0 & 0 \\
1 & 1 & 0 & 1 \\
\hline
\end{tabular}

Triad 1 can serve as a demultiplexer by virtue of the fact that it can exist in various isomeric states. The synthesis and structure of $\mathbf{1}$ have been reported. ${ }^{8}$ The triad consists of a tetraarylporphyrin (P) linked to both a substituted dihydropyrene photochrome (DHP) ${ }^{35}$ and a photochrome of the dihydroindolizine family ${ }^{36}$ based on pyrrolo[1,2-b]pyridazine (DHI). Because each photochrome may exist in two metastable forms, the triad may assume any of four isomeric structures (ignoring chirality). Although the three chromophores of $\mathbf{1}$ interact photochemically (vide infra), the linkages joining them have been designed so that photoisomerization of the two photochromes still occurs, and it is possible to prepare photostationary distributions greatly enriched in any of the four isomers. ${ }^{8}$ For the demultiplexer, only the three isomers shown in Figure 1 are required.

\section{Results and Discussion}


Photochemistry of the triad. Here, we briefly summarize the photochemistry of the two photochromic molecules, and then present results for triad 1. In general, DHI-type photochromes do not absorb visible light, are thermally stable, and can be photoisomerized to an open, visible-absorbing betaine form (BT) using ultraviolet light (Figure 1). With $366 \mathrm{~nm}$ radiation, for example, the resulting photostationary distribution is 15:85 DHI:BT. ${ }^{4}$ The BT isomer thermally reverts back to essentially pure DHI with a time constant of $4000 \mathrm{~s}$ at $29{ }^{\circ} \mathrm{C} .{ }^{8}$ At $55^{\circ} \mathrm{C}$, thermal reversion is complete after $30 \mathrm{~min}$.

The DHP photochrome is the thermally most stable isomer, and absorbs visible light. Irradiation with green light (e.g. $532 \mathrm{~nm}$ ) photoisomerizes DHP to the CPD form (Figure 1) with 100\% efficiency $(\Phi=10 \%){ }^{3}$ The CPD form can be isomerized back to DHP with heat $\left(\tau=27 \mathrm{~h}\right.$ at $\left.25^{\circ} \mathrm{C}\right) .^{3}$ Irradiation of CPD with UV light also returns it to the DHP form $(\Phi=30 \%)$, and with wavelengths $<300 \mathrm{~nm}$, the photostationary distribution is highly enriched in DHP. However, this photoisomerization was not employed in the demultiplexer experiments. Both photochromes undergo relatively little $(<3 \%$ per cycle) photodecomposition when solutions are rigorously degassed. In the presence of oxygen, the DHI is especially sensitive to photodecomposition.

The absorption spectra of these three isomers and a model porphyrin are shown in Figure 2. As revealed below, the changes in the absorption spectra upon photoisomerization comprise one output of the demultiplexer $(\mathrm{O} 2)$. Isomer DHP-P-DHI is the thermally stable form of the molecule. As shown in Figure 2, the absorption spectrum of this isomer in 2-methyltetrahydrofuran in the visible region features bands similar to those of a model porphyrin, 5,15-bis(4-methoxycarbonylphenyl)-10,20bis(2,4,6-trimethylphenyl)porphyrin, (418 (Soret), 515, 549, 592 and $649 \mathrm{~nm}$ ), underlain by a broad absorption band of the DHP moiety at $\sim 513 \mathrm{~nm}$. The DHP and DHI moieties also absorb in the $300-$ $400 \mathrm{~nm}$ region. Irradiation of the solution of DHP-P-DHI with green light (532 nm) converts the DHP component of $\mathbf{1}$ to the cyclophanediene (CPD) form, generating a photostationary state containing mainly CPD-P-DHI. The absorption spectrum of CPD-P-DHI is very similar to that of the model porphyrin in the visible region and features absorbance characteristic of the photochromes mainly in the 
ultraviolet (Figure 2). ${ }^{8}$ The absorbance in the $300-400 \mathrm{~nm}$ region is significantly reduced relative to that of DHP-P-DHI due to the absence of the contribution from DHP.

Irradiating DHP-P-DHI with UV light $(355 \mathrm{~nm})$ under the conditions described in the Experimental Section converts it to DHP-P-BT, where the DHI chromophore has photoisomerized to the open-chain, zwitterionic betaine form (BT). (Under these conditions, photoisomerization of DHP to CPD is relatively minor.) The absorption spectrum of DHP-P-BT resembles that of DHP-P-DHI in the $300-$ $400 \mathrm{~nm}$ region, but the strong, broad absorbance of the betaine in the $440-650 \mathrm{~nm}$ range $\left(\lambda_{\max } \sim 560\right.$ nm) now dominates the visible region (Figure 2).

The other output of the demultiplexer $(O 1)$ is fluorescence from the porphyrin. Because the porphyrin and the two photochromes can communicate with one another due to intramolecular energy and electron transfer phenomena, the amplitude of this fluorescence is a function of the structures of the two attached photochromes (Figure 3). The shapes of the emission spectra of all of the isomers of $\mathbf{1}$ are similar to that of the model porphyrin, with maxima at $651 \mathrm{~nm}$ and $720 \mathrm{~nm}$. The quantum yield of emission for CPDP-DHI is essentially the same as that for the porphyrin model compound, as the CPD and DHI moieties do not affect the porphyrin. The lifetime of the porphyrin first excited singlet state of this isomer is 11 $\mathrm{ns},{ }^{8}$ which is similar to that of the first excited singlet state of the model porphyrin. Conversion to DHPP-DHI results in significant quenching of the porphyrin fluorescence (Figure 3). The lifetime of DHP${ }^{1} \mathrm{P}-\mathrm{DHI}$ is $1.55 \mathrm{~ns}$. This quenching has been ascribed to photoinduced electron transfer to form a shortlived DHP ${ }^{\bullet+}-\mathrm{P}^{\bullet-}$-DHI charge-separated state. ${ }^{8}$ Even stronger quenching is observed in DHP-P-BT. In this isomer, the porphyrin first excited singlet state is also quenched by very rapid photoinduced electron transfer to the betaine to form a charge-separated state DHP-P ${ }^{\bullet+}-\mathrm{BT}^{\bullet-}$. The rate constant for this quenching, based on a model dyad, is $2.0 \times 10^{10} \mathrm{~s}^{-1.8}$

Demultiplexer function. Triad 1 can function as a 1:2 demultiplexer in combination with pulsed laser inputs and a third harmonic generating (THG) crystal. The design concept is shown in Figure 4. Triad 1 in 2-methyltetrahydrofuran solution resides in a temperature-controlled cuvette. Input In, light at 532 
$\mathrm{nm}$, is provided by a laser. A convenient source is a Nd:YAG laser at $1064 \mathrm{~nm}$ and a second harmonic generating crystal (SHG). The address input, $A d$, comes from the first harmonic of a second Nd:YAG laser at $1064 \mathrm{~nm}$. Both laser beams pass through a THG crystal placed in front of the sample cuvette. Output $O 1$ is porphyrin fluorescence monitored at a convenient wavelength (e.g. $720 \mathrm{~nm}$ ) and output $O 2$ is the sample absorbance at $572 \mathrm{~nm}$. In the experimental realization of this device detailed below, only a single laser was used for reasons of convenience, and the SHG and THG crystals were tuned on or off resonance to achieve the proper input wavelengths, as discussed in the Experimental Section.

The operation of the demultiplexer begins with a reset operation, consisting of heating the sample for $3 \mathrm{~h}$ at $55^{\circ} \mathrm{C}$. This converts triad 1 into its thermally stable DHP-P-DHI form. Under these conditions, with neither input on, porphyrin fluorescence at $720 \mathrm{~nm}$ is weak $(O 1=0)$, because the DHP chromophore quenches porphyrin emission, as discussed above. The absorbance at $572 \mathrm{~nm}$ is also low $(O 2=0)$ because the concentration of the betaine, which has the largest extinction coefficient at this wavelength, is zero, or very low. Experimental results for this situation (corresponding to the first line in Table 1) are shown in Figure 5. Output $O 1$ is shown in the first column of Figure 5a, and output $O 2$ appears in the first column of Figure 5b. The dashed lines represent a threshold level; a signal above the threshold represents an on response. Any real digital device (including transistors) must employ a threshold to allow a distinction between signal and noise.

When input In is switched on, the 532-nm radiation causes the DHP photochrome to rapidly isomerize to the CPD form. Isomerization of DHI to BT under these conditions is much slower. Thus, the sample is converted mainly to CPD-P-DHI. In this isomer, porphyrin fluorescence is strong, as no quenching occurs $(O 1=1)$, but absorbance at $572 \mathrm{~nm}$ is still weak $(O 2=0)$. The conditions of the second row of the truth table are fulfilled (Figure 5).

Alternatively, if the address input $A d$ is turned on by exposing the sample to $1064 \mathrm{~nm}$ light, the triad will remain in the DHP-P-DHI form, and both $O 1=0$ and $O 2=0$, corresponding to row three of Table 1. The response under these conditions is also shown in Figure 5. 
The final row of the truth table corresponds to turning on both the $532 \mathrm{~nm}$ input (In) and the $1064 \mathrm{~nm}$ address input $(A d)$. Under these conditions, $355 \mathrm{~nm}$ light is generated in the THG crystal. These conditions isomerize DHI to BT, but have relatively little effect on DHP, so the sample is converted mainly to DHP-P-BT. The sample now has only weak porphyrin emission at $720 \mathrm{~nm}$ (Figure 5) due to quenching by both DHP and BT. However, the absorbance at $572 \mathrm{~nm}$ is now quite large, due to the BT moiety. Output $O 1$ is off, but $O 2$ is on.

Thus, the response of the system is that of a 1:2 digital demultiplexer. When $1064 \mathrm{~nm}$ address input $A d$ is off, output $O 2$ is also off, and $O 1$ tracks the state of input In. When $A d$ is turned on, output $O 1$ remains off, and $O 2$ reports the state of $I n$.

Cycling and signal-to-noise ratio. The intent of this work was to demonstrate the basic principles involved, and not to prepare a technologically useful device. No attempt was made to optimize performance or stability. However, we did monitor the system through several cycles, as illustrated in Figure 6. Figure 6a shows the emission intensity at $720 \mathrm{~nm}(O 1)$ following combinations of the various operations: reset $(R), \operatorname{In}=1(\operatorname{In}), A d=1(A d)$. The corresponding results for $O 2$ (absorbance at $572 \mathrm{~nm})$ appear in Figure 6b. These data also demonstrate the high signal-to-noise levels of the two outputs. The noise levels are very low relative to the changes in emission intensity and absorbance resulting from the switching operations.

\section{Conclusions}

The results above demonstrate that triad $\mathbf{1}$ can function as a 1:2 digital demultiplexer when coupled to a THG crystal. In this device, the two photochromes serve to respond to the laser inputs and record the state of each input applied for later readout. The porphyrin moiety "interrogates" the photochromes via electron transfer phenomena, correlates their states, and provides an output consistent with the demultiplexer function. The THG crystal is an integral part of the demultiplexer because it allows the molecule to respond in the correct way to the simultaneous application of both inputs. The use of this approach in an actual technologically useful device would in principle be possible if the photo- and 
thermal stability of the triad and other inherent features of the system were compatible with the intended use. Obvious refinements could include containment of the triad within a polymer film located behind the THG crystal and use of spatially sensitive output detectors so that multiple demultiplexers could be housed in a single unit. In this connection, it should be noted that any applications need not be limited to replacement of functions currently carried out by conventional electronics. Molecule-based logic elements can in principle be employed in configurations (e.g. nanoscale particles or "containers") and environments (e.g., aqueous and biological) where conventional devices might be less well suited.

Triad $\mathbf{1}$ is an excellent illustration of the fact that a single photonically operated, molecule-based logic system can be reconfigured for a variety of tasks simply by changing the initial state and the nature of the inputs. It is not necessary to physically alter the medium containing the molecules, or to access it with anything other than light. Thus, 1 can be converted from a 1:2 demultiplexer to a 2:1 multiplexer, and AND gate, or an INHIBIT gate, depending on the optical setup. This flexibility of molecule-based photonic systems may make them valuable for future applications where a device must be rapidly reconfigured for multiple functions.

Switching speeds are not practically limited by the rates of the photoisomerization reactions, as these occur on the ps - ns time scales. Rather, the switching times are controlled by the light flux from the input lasers, and by the temperature for the thermal reset operation (which is rather slow at $55{ }^{\circ} \mathrm{C}$ ). Higher light levels and temperatures would obviously speed up operation (until stability limits were reached).

In order to make our previously-reported multiplexer ${ }^{34}$ function in concert with the 1:2 demultiplexer, additional circuitry would be needed. The output of the multiplexer (porphyrin fluorescence) would have to be converted to drive the demultiplexer In input (532 nm light), and the control elements (532 nm light for the multiplexer and $1064 \mathrm{~nm}$ light for the demultiplexer) would have to be driven in phase with one another.

\section{Experimental Section}


The synthesis and characterization of triad $\mathbf{1}$ and its basic photochemistry have been reported previously. ${ }^{8}$ Distilled 2-methyltetrahydrofuran was used as the solvent for spectroscopic measurements, and the sample concentration was $5 \times 10^{-6} \mathrm{M}$. The samples were degassed by six freeze-pump-thaw cycles to a final pressure of approximately $10^{-5}$ Torr. The absorption measurements were performed using a CARY 4B UV/vis spectrometer. A SPEX Fluorolog $\tau 2$ was used for the emission measurements. After exposure to the different input combinations, the absorbance and the emission were monitored separately using the instruments described above. Inputs were provided by a Nd:YAG laser (Continuum Surelight II-10, $6 \mathrm{~ns}$ fwhm). The samples were stirred continuously during all irradiation processes. Only a single laser was available to generate both inputs. As a result, the state with the Ad input only on was generated by passing the first harmonic of the laser $(1064 \mathrm{~nm}, 10 \mathrm{~Hz}, 340 \mathrm{~mW}$ average power, $35 \mathrm{~s}$ ) through the two non-linear crystals with the SHG crystal tuned off-resonance and the THG crystal tuned in-resonance. The state with the In input only on was generated by tuning the SHG crystal in-resonance $(532 \mathrm{~nm}, 10 \mathrm{~Hz}, 20 \mathrm{~mW}$ average power, $35 \mathrm{~s}$ ) and the THG crystal offresonance. When both crystals were in-resonance, (state Ad on and In on) $355 \mathrm{~nm}$ UV light (10 Hz, 12 $\mathrm{mW}$ average power, $35 \mathrm{~s}$ ) was generated by the THG crystal. In this configuration, the crystals could not be set completely to $0 \%$ harmonic generation. In order to maximize the degree of discrimination among the isomer populations, a $532 \mathrm{~nm}$ dichroic mirror was used to better eliminate the UV light from the 532-nm light (input In on), and to eliminate the 532-nm light from the IR light (input Ad on). No filter was used when both inputs were on, i.e., when both the SHG and the THG were tuned in-resonance to generate UV light. Had two lasers been available, the use of this mirror and crystal tuning could have been avoided. Various alternative setups, such as using a single laser and controlling the wavelength reaching the THG and sample with bandpass filters, or using a single laser with a split beam and highspeed electronically switched shutters, can be envisioned.

Acknowledgment. This work was supported by the National Science Foundation (CHE-0352599) and the Swedish Research Council, VR. RHM thanks NSERC of Canada for financial support. 
1. Kuciauskas, D.; Liddell, P. A.; Moore, A. L.; Moore, T. A.; Gust, D. J. Am. Chem. Soc. 1998, $120,10880-10886$.

2. Liddell, P. A.; Kodis, G.; Moore, A. L.; Moore, T. A.; Gust, D. J. Am. Chem. Soc. 2002, 124 , 7668-7669.

3. Liddell, P. A.; Kodis, G.; Andréasson, J.; de la Garza, L.; Bandyopadhyay, S.; Mitchell, R. H.; Moore, T. A.; Moore, A. L.; Gust, D. J. Am. Chem. Soc. 2004, 126 , 4803-4811.

4. Straight, S. D.; Andréasson, J.; Kodis, G.; Moore, A. L.; Moore, T. A.; Gust, D. J. Am. Chem. Soc. 2005, $127,2717-2724$.

5. Straight, S. D.; Terazono, Y.; Kodis, G.; Moore, T. A.; Moore, A. L.; Gust, D. Aust. J. Chem. 2006, $59,170-174$.

6. Terazono, Y.; Kodis, G.; Andréasson, J.; Jeong, G.; Brune, A.; Hartmann, T.; Dürr, H.; Moore, A. L.; Moore, T. A.; Gust, D. J. Phys. Chem. B 2004, 108 , 1812-1814.

7. Bahr, J. L.; Kodis, G.; de la Garza, L.; Lin, S.; Moore, A. L.; Moore, T. A.; Gust, D. J. Am. Chem. Soc. 2001, 123 , 7124-7133.

8. Straight, S. D.; Andréasson, J.; Kodis, G.; Bandyopadhyay, S.; Mitchell, R. H.; Moore, T. A.; Moore, A. L.; Gust, D. J. Am. Chem. Soc. 2005, 127 , 9403-9409.

9. Straight, S. D.; Liddell, P. A.; Terazono, Y.; Moore, T. A.; Moore, A. L.; Gust, D. Adv. Funct. Mater. 2007, 17, 777-785. 
10. Andréasson, J.; Terazono, Y.; Albinsson, B.; Moore, T. A.; Moore, A. L.; Gust, D. Angew. Chem. Int. Ed. 2005, $44,7591-7594$.

11. Andréasson, J.; Straight, S. D.; Kodis, G.; Park, C.-D.; Hambourger, M.; Gervaldo, M.; Albinsson, B.; Moore, T. A.; Moore, A. L.; Gust, D. J. Am. Chem. Soc. 2006, 12 , 16259-16265.

12. Andréasson, J.; Kodis, G.; Terazono, Y.; Liddell, P. A.; Bandyopadhyay, S.; Mitchell, R. H.; Moore, T. A.; Moore, A. L.; Gust, D. J. Am. Chem. Soc. 2004, 126 , 15926-15927.

13. Gust, D.; Moore, T. A.; Moore, A. L. Chem. Commun. 2006, 2006 , 1169-1178.

14. Pina, F.; Melo, M. J.; Maestri, M.; Passaniti, P.; Balzani, V. J. Am. Chem. Soc. 2000, 122 , 4496-4498.

15. Langford, S. J.; Yann, T. J. Am. Chem. Soc. 2003, 125 , 11198-11199.

16. Gunnlaugsson, T.; Mac Donaill, D. A.; Parker, D. J. Am. Chem. Soc. 2001, 123 , 12866-12876.

17. Ghosh, P.; Bharadwaj, P. K. J. Am. Chem. Soc. 1996, 118 , 1553-1554.

18. de Silva, A. P.; McClenaghan, N. D. Chem. Eur. J. 2002, 8, 4935-4945.

19. Xu, H.; Xu, X.; Dabestani, R.; Brown, G. M.; Fan, L.; Patton, S.; Ji, H. F. J. Chem. Soc. ,Perkin Trans. 2002, 2 , 636-643.

20. Raymo, F. M.; Giordani, S. J. Am. Chem. Soc. 2002, 124 , 2004-2007.

21. Saghatelian, A.; Volcker, N. H.; Guckian, K. M.; Lin, V. S. Y.; Ghadiri, M. R. J. Am. Chem. Soc. 2003, 125, 346-347.

22. Kompa, K. L.; Levine, R. D. Proc. Natl. Acad. Sci. 2001, 98 , 410-414.

23. Guo, X.; Zhang, D.; Zhang, G.; Zhu, D. J. Phys. Chem. B 2004, 108, 11942-11945. 
24. Margulies, D.; Melman, G.; Shanzer, A. Nature Materials 2005, 4, 768-771.

25. Margulies, D.; Melman, G.; Felder, C. E.; Arad-Yellin, R.; Shanzer, A. J. Am. Chem. Soc. 2004, $126,15400-15401$.

26. Zhou, Y.; Wu, H.; Qu, L.; Zhang, D.; Zhu, D. J. Phys. Chem. B 2006, 110 , 15676-15679.

27. Pischel, U. Angew. Chem. Int. Ed. 2007, 46 , 4026-4040.

28. Niazov, T.; Baron, R.; Katz, E.; Lioubashevski, O.; Willner, I. Proc. Nat. Acad. Sc. U. S. A. 2006, $103,17160-17163$.

29. Suresh, M.; Jose, D. A.; Das, A. Org. Lett. 2007, 9 , 441-444.

30. Remacle, F.; Weinkauf, R.; Levine, R. D. J. Phys. Chem. A 2006, 110, 177-184.

31. Liu, Y.; Jiang, W.; Zhang, H. Y.; Li, C. J. J. Phys. Chem. B 2006, 110, 14231-14235.

32. Margulies, D.; Felder, C. E.; Melman, G.; Shanzer, A. J. Am. Chem. Soc. 2007, 129, 347-354.

33. Szacilowski, K. Chem.Eur. J. 2004, 10 , 2520-2528.

34. Andréasson, J.; Straight, S. D.; Bandyopadhyay, S.; Mitchell, R. H.; Moore, T. A.; Moore, A. L.; Gust, D. Angew. Chem. Int. Ed. 2007, 46 , 958-961.

35. Mitchell, R. H. Euro. J. Org. Chem. 1999, 1999, 2695-2703.

36. Dürr, H. Photochromism of dihydroindolizines and related systems. In Organic Photochromic and Thermochromic Compounds, 1 ed.; Crano, J. C., Guglielmetti, R. J., Eds.; Plenum Press: New York, 1999; pp 223-266. 
Figure Captions

Figure 1. Three photoisomers of triad 1 and relevant interconversion pathways. A fourth isomer is not relevant for use of $\mathbf{1}$ in the demultiplexer, and the structure is not shown. The experimental conditions for the isomerizations are given in the text.

Figure 2. Absorption spectra in 2-methyltetrahydrofuran of pure or highly enriched solutions of 5,15bis(4-methoxycarbonylphenyl)-10,20-bis(2,4,6-trimethylphenyl)porphyrin (- $(-)$ the DHP-P-DHI isomer of $1(----)$, CPD-P-DHI $(-\bullet-\bullet-\bullet)$ and DHP-P-BT $(\bullet \bullet \bullet \bullet)$.

Figure 3. Emission spectra with $590 \mathrm{~nm}$ excitation in 2-methyltetrahydrofuran: 5,15-bis(4methoxycarbonylphenyl)-10,20-bis(2,4,6-trimethylphenyl)porphyrin ( - ) (arbitrary amplitude), the DHP-P-DHI isomer of $1\left(--_{-}\right)$, and photostationary states of the same sample highly enriched in

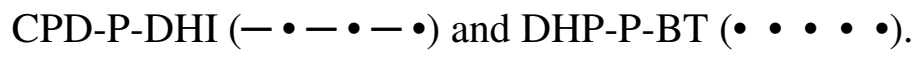

Figure 4. Conceptual diagram of the 1:2 demultiplexer system. Triad $\mathbf{1}$ is dissolved in 2methyltetrahydrofuran and placed in a cuvette. Input $A d$ is $1064 \mathrm{~nm}$ light from a pulsed Nd:YAG laser. Input In is $532 \mathrm{~nm}$ light provided by light from a similar laser passing through a SHG crystal. The third harmonic generating (THG) crystal does not affect the 1064 or $532 \mathrm{~nm}$ beams, but when both are present, generates $355 \mathrm{~nm}$ light. The readout sources provide light for measurement of the porphyrin fluorescence emission at $720 \mathrm{~nm}(O 1)$ and the betaine absorbance at $572 \mathrm{~nm}(O 2)$. In practice, both laser beams were generated by the same laser (see Experimental Section).

Figure 5. Experimental response levels for the outputs. The dashed horizontal lines represent threshold levels for distinguishing between on and off responses. (a) Porphyrin fluorescence emission intensity (arbitrary scale) at $720 \mathrm{~nm}$. (b) Absorbance (due mainly to betaine) at $572 \mathrm{~nm}$. The horizontal scales indicate the states $(1=$ on, $0=$ off $)$ of data input $I n$ and address input $A d$.

Figure 6. Cycling of the triad 1 after sequential exposure to the various input combinations. Symbol $R$ indicates the reset operation, In indicates exposure to $532 \mathrm{~nm}$ irradiation, and Ad signifies $1064 \mathrm{~nm}$ 
exposure. (a) Emission intensity at $720 \mathrm{~nm}$ (arbitrary units) (b) Absorbance at $572 \mathrm{~nm}$. The traces are the actual measured signals, and therefore demonstrate the signal-to-noise levels present during the measurements. 
Figure 1

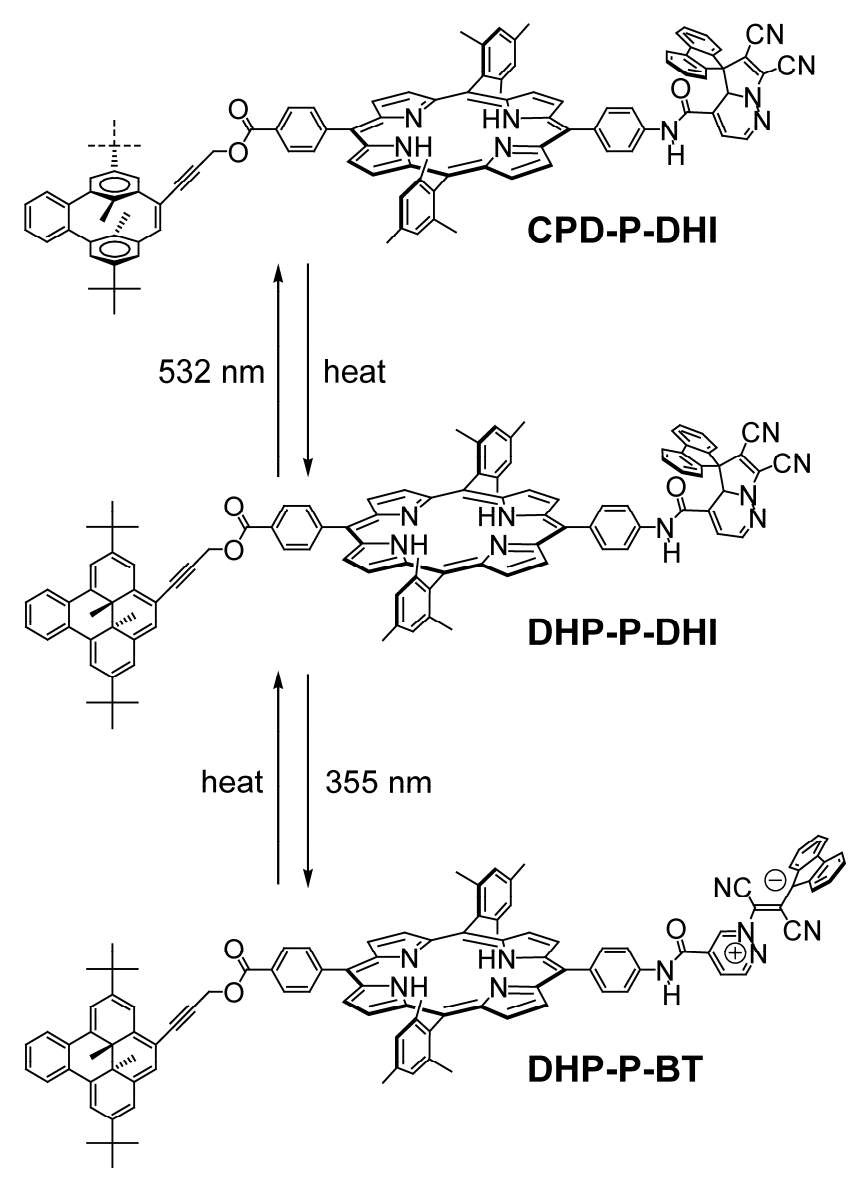


Figure 2

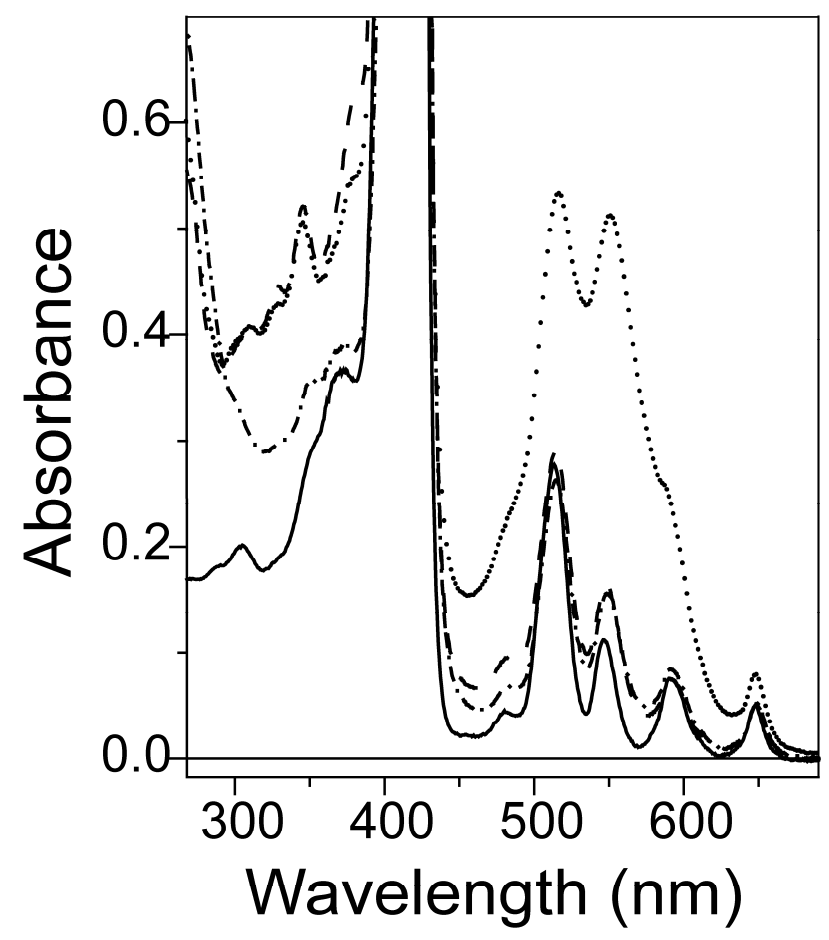


Figure 3

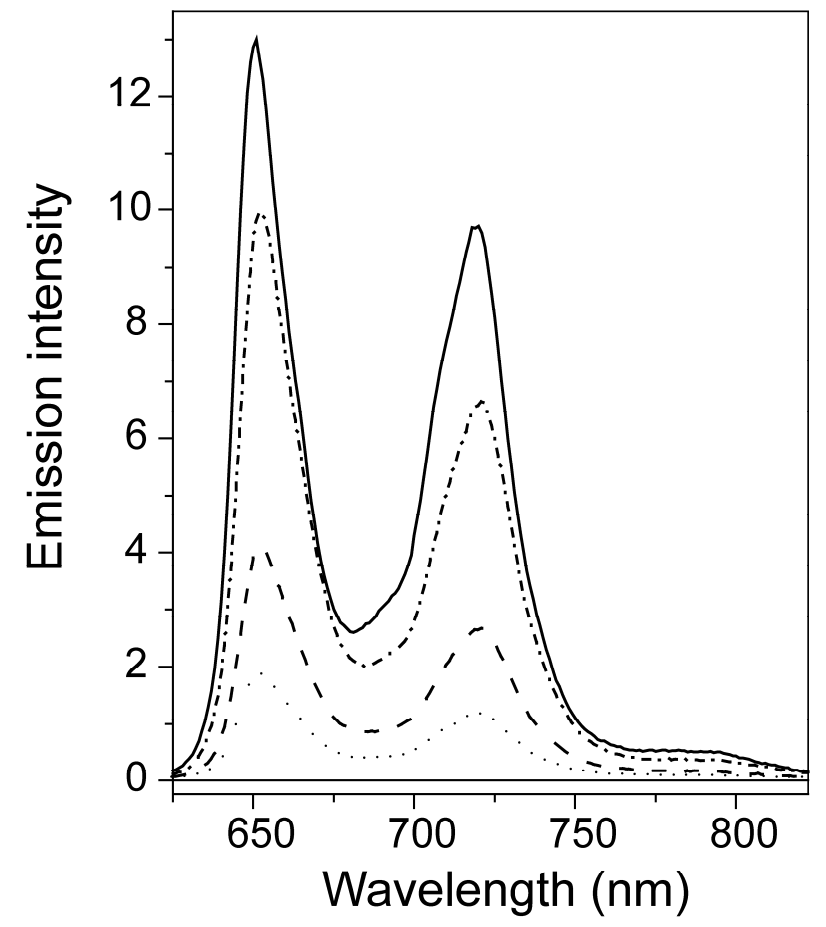


Figure 4

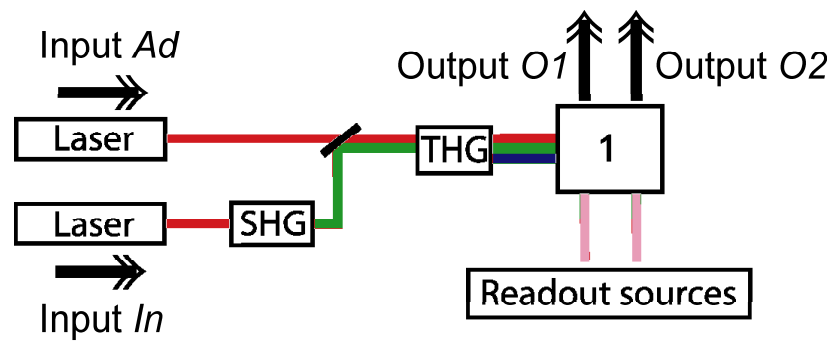


Figure 5
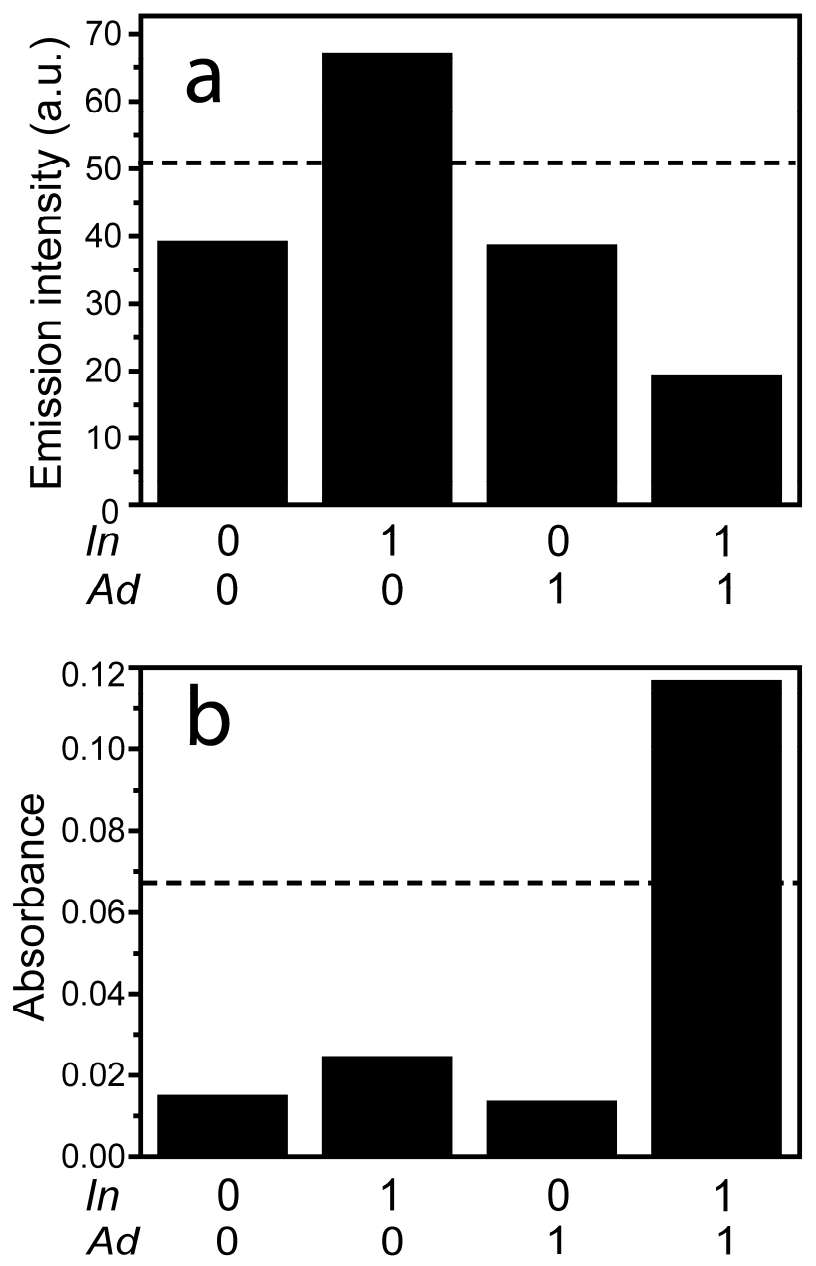
Figure 6
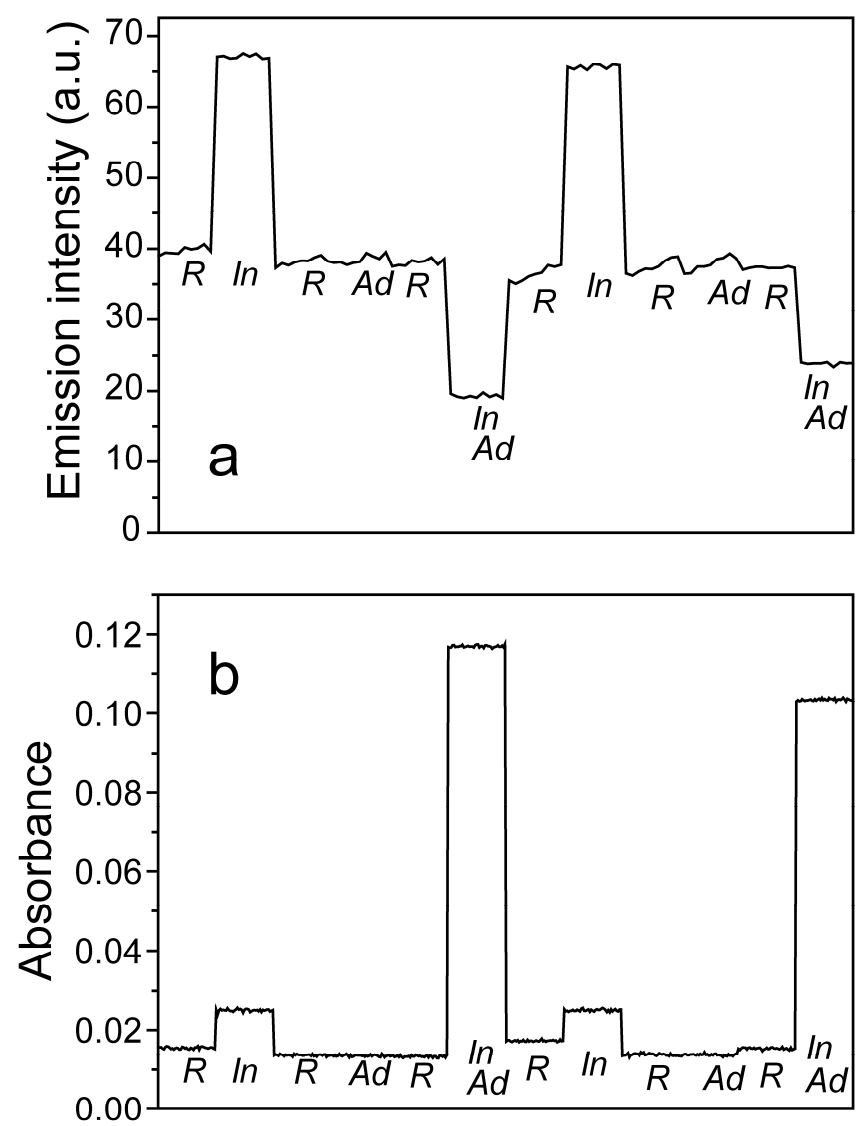